\title{
LSI BASED SEARCH TECHNIQUE: USING EXTRACTED KEYWORDS AND KEY-SENTENCES
}

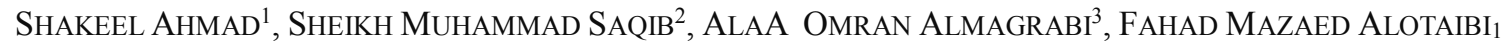 \\ ${ }^{1}$ Faculty of Computing and Information Technology in Rabigh (FCITR) King Abdul Aziz University (KAU) Jeddah \\ Saudi Arabia \\ ${ }^{2}$ Institute of Computing and Information Technology Gomal University. Pakistan. \\ ${ }^{3}$ Department of Information Systems, Faculty of Computing and Information Technology (FCIT) King Abdul Aziz \\ University (KAU) Jeddah Saudi Arabia \\ ${ }^{1}$ sarahmad@kau.edu.sa, ${ }^{1}$ fmmalotaibi@kau.edu.sa,${ }^{2}$ saqibsheikh4@gu.edu.pk, ${ }^{3}$ aalmagrabi3@kau.edu.sa
}

\section{Revised November 2017}

\begin{abstract}
Now a day bulk of information available on Internet but in spite of will, no one can go through all these information, which may carry huge amount of data. Hence, most of the web surfer required a mechanism through which particular documents or sentences of their interests can be extracted easily without wasting much amount of time. For this purpose, we can generate some keywords out of the stored information, so that keywords requested by end user should match easily with stored keywords to detect sentences. In this context, Latent Semantic Indexing (LSI) based search method has been applied on whole text and keys. It has been observed through the results that the method with keys (Keywords and key-Sentences) found considerably proficient. Moreover, extracted Keys are not only suited for searching but also appropriate for clustering. Finally, the proposed study not only investigated the detection of keywords but also involved exposure of key sentences.
\end{abstract}

Keywords:- Latent semantic indexing (LSI), opinion mining, sentiment analysis, sentiment classification, supervised machine learning, singular value decomposition (SVD).

1. Introduction: In opinion mining field, when we want to analyze any document i.e. summary of document or sentiment analysis of document, besides all other efforts first of all it is very important to find out that either selected document belongs to required key words or aspects or entities. Most of the work has been done by [1][2][3] related to aspect-based analysis. Support Vector Machine SVM can recognize and analyze the aspects for sentiment classification [16], Supervised Machine Learning algorithm focuses on mining relevant information from reviews has been done by [17][18] related to aspect based sentiment analysis. The work done by [19] has shown the hybrid classification for aspect-based classification. To check whether, selected document is relevant to required aspect; first of all we will extract aspects from selected document and then compare those aspects with required aspects. How we can extract such aspects, there is lot of methods in literature proposed by different researchers. Study of [4] has proposed a novel rule-based approach to extract an aspect from reviews of product. This paper [5] has presented an unsupervised approach to find out polarity of an aspect from different domains. In [6][10][11] summarization of aspects with respect to sentiment score has been proposed. Some researchers has explored aspect from a document with respect to subjective words [12][13] means a words which have no polarity can be considered as an aspect. A machine learning technique has been used to find out rating estimation of an aspect for reviews. Information distance was the asset for such rating [7]. Researchers have extended the aspect-based opinion mining of Bing Liu's [8] which was for physical products. This [9] extended work has used more complex Natural Language Processing- NLP based rules for online tourism product reviews. A supervised learning algorithm has been used to find out important aspects and predictions with respect to reviews of such item [14]. Logic Programming, particularly Answer Set Programming 
(ASP), has been used to elegantly and efficiently implement the key components of syntax-based aspect extraction [15]. Aspect detection is more important, so it must be identified accurately. Besides detection of aspects there is also lot of work based on detection of key words. LSI (Latent Semantic Indexing) method is better for searching, it has been used in [23] for the clustering of documents and for concept representations. An extended method based on LSI is able to filter unwanted emails in Chinese and English [36]. A framework using the LSI method with a little bit of pre-processing work has used to determine the polarity of a review [25]. Term extraction is a process of recognizing the important entities in a specific domain and it often relies on domain-specific knowledge in order to upgrade and improve the system performance. Keyword extraction is also process of identifying a short list of words or noun phrases that capture the most important ideas or topics covered in a document [7]. This study investigates a technique to extract keywords and key sentences from text which is very helpful in clustering, making datasets etc. keywords and key-sentences with text document as metadata, through which searching process will be easy and fast. Then from any searched query, keywords can be detected and will find score using LSI with already stored keywords of different documents to detect closest information.

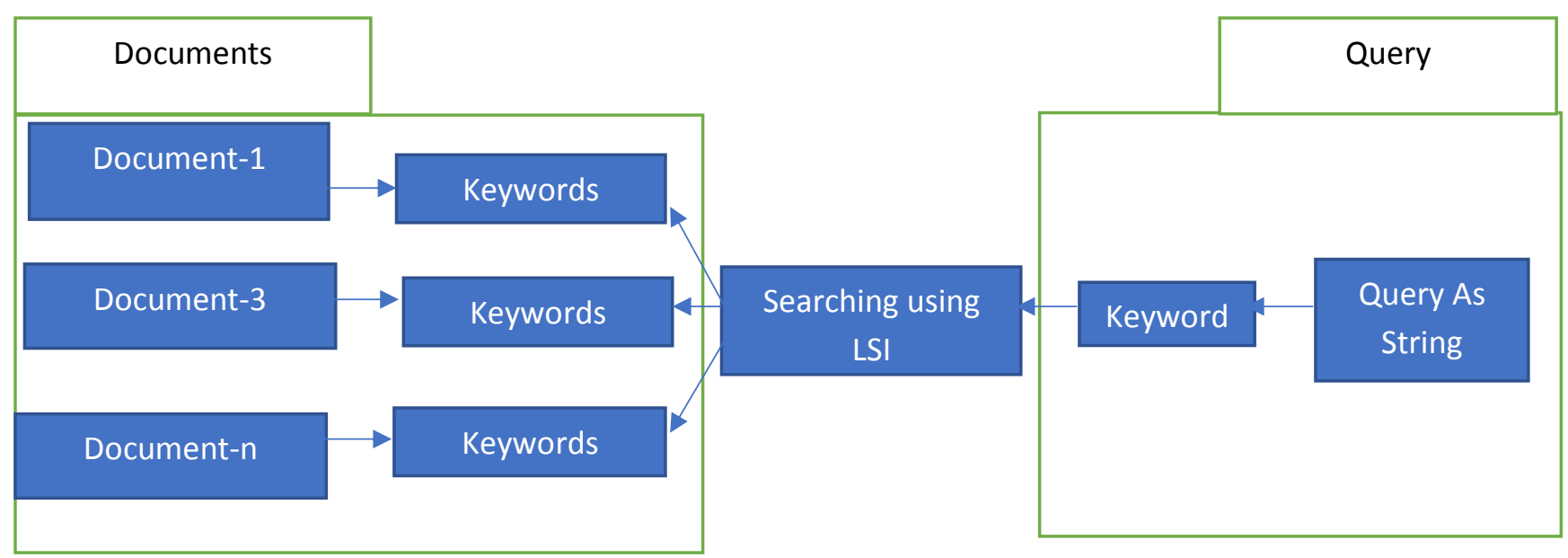

Fig-1. Searching Mechanism of Proposed Work Based on Keywords.

This study made the following key contributions:

- A method was proposed for Keywords, Key-Sentences and No-Word for searching and clustering purpose, using similarity path distance of WordNet dictionary.

- Determine closest document with given query with the help of extracted Key-Words and Key-Sentence by using LSI.

- Compare the LSI based search with whole documents and with keys.

2. Extraction of Keys: Proposed work will detect the keywords and key sentences based on similarity distance of words using WordNet dictionary. Proposed work is depicted in Fig-2. In this figure, a sentence consist of 12 words, where key-sentences, keywords and No-words have been created. A word which have a similarity distance greater than 0.6 with next word is appended with a string. Now next appended word will determine distance with its adjacent next word, this next word will be appended with string if distance is greater than 0.6. When distance will less than 0.6, then stop to append with string. Now this string can be considered as keyword as it has two words, if there are more than two words in a string, then it can be considered as key-sentence. 


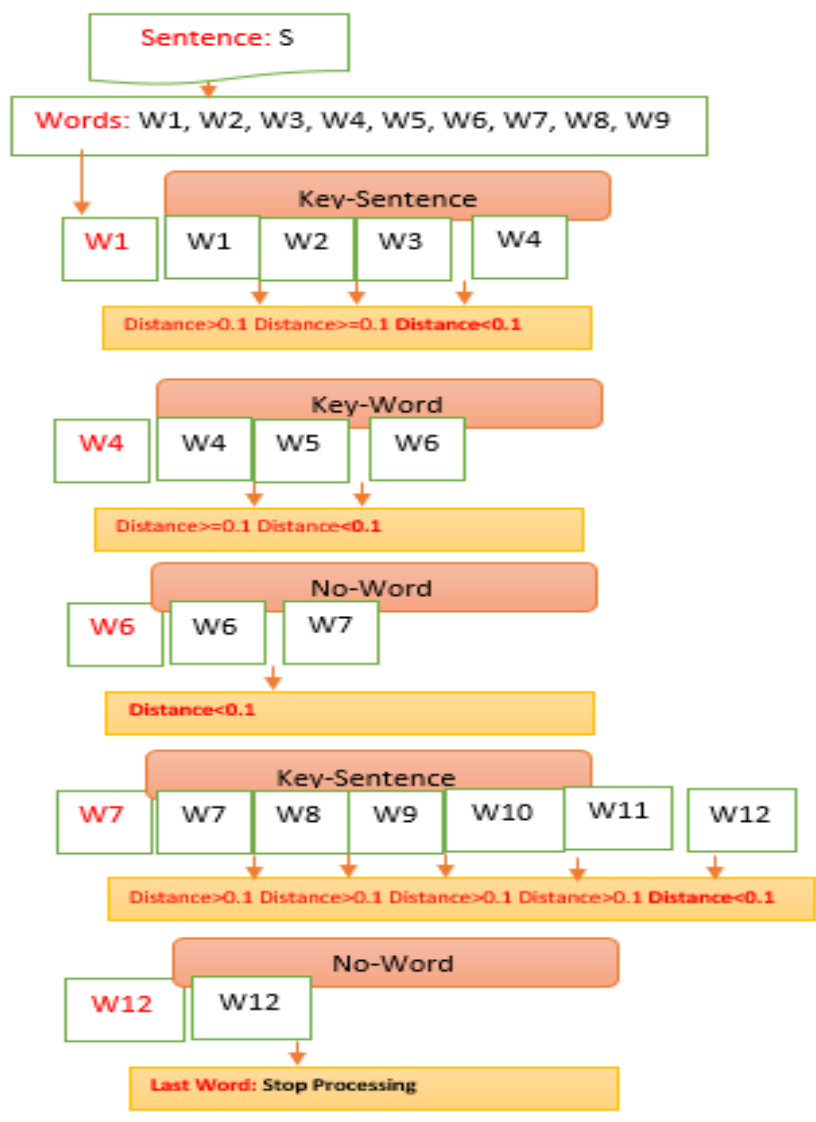

Fig-2. Steps of Proposed Work

In above figure, word w1 has found distance with w2 which is greater than 0.1 , both will be appended in key-sentence. Now w2 found distance with w 3 which is also greater than 0.1 means w 3 will be added. Then w3 found distance with w4 which is less than 0.1, stop this process. Key sentence has three words "w1 w2 w3". Now algorithm will determine next word from w4. As w4 and w5 distance is greater than 0.1 while w6 distance with w5 is not greater than six, hence it is keyword consist of "w4 w5". Now w6 has less than 0.6 distance from w7, so there is no keyword, which can be considered as No-word.

3. Searching through LSI: LSI, which was proposed by Deerwester in 1990, is an efficient information retrieval algorithm [24]. Basically, in LSI, there is a cosine similarity measure between the coordinates of a document vector and the coordinates of a query vector. If this value is 1 , it means the document is $100 \%$ closer to the query, if it is 0.5 , it means the document is $50 \%$ closer to the query, and if it is 0.9 , it means the document is $90 \%$ closer to the query.

The major point now is finding the coordinates of each document and query. A singular value decomposition (SVD) can determine the points or coordinates of a document and query. Through the SVD, three matrices, S, V and $\mathrm{U}$, which will be used for further processing, can be determined by a matrix. To determine the values of such variables, the SVD requires a matrix. The matrix consists of rows and columns containing integers, but the inputs under consideration are the different text documents. A feature matrix can be obtained by calculating the frequencies of each word. This means that first, a feature matrix is created from all the documents, and then, the SVD is calculated. After this, the supporting variables, S, V and U, will be calculated by using NumPy (Numeric Python). The coordinates of all the documents will be determined from $\mathrm{S}$, and these coordinates will be merged with the query to obtain the query coordinates. Finally, a cosine similarity function will be applied to these coordinates to find the documents that are closest to the query [23]. 


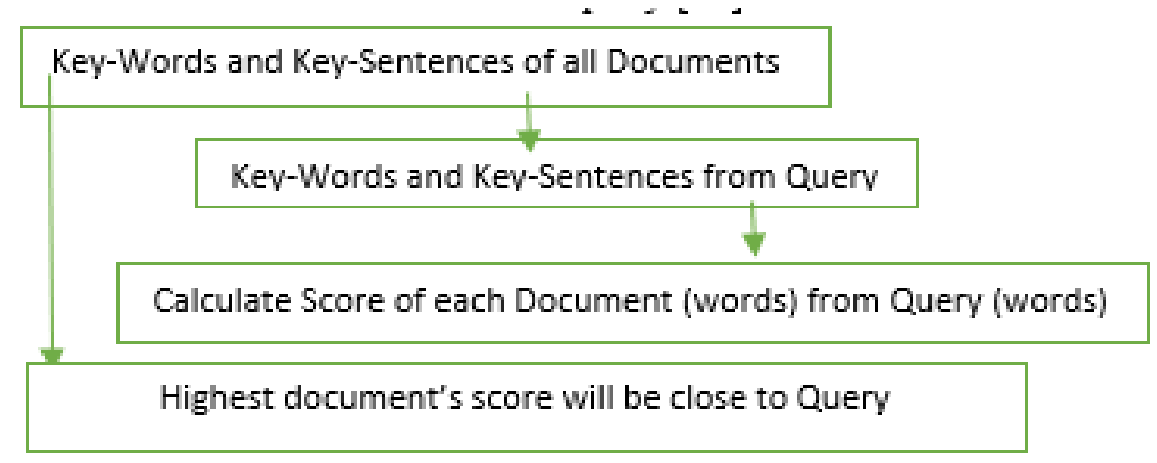

Fig-3. LSI Based Search using Keys.

In Table-1, fWords is the input list containing filtered words i.e. there is no stop words, all in lower case. KeyW is the out list contain Key-Sentence, Key-Words and No-Words. Used path distance between two words is path_similarity, which taken from the synset dictionary of wordnet.

Table-1: Algorithm of Proposed Model

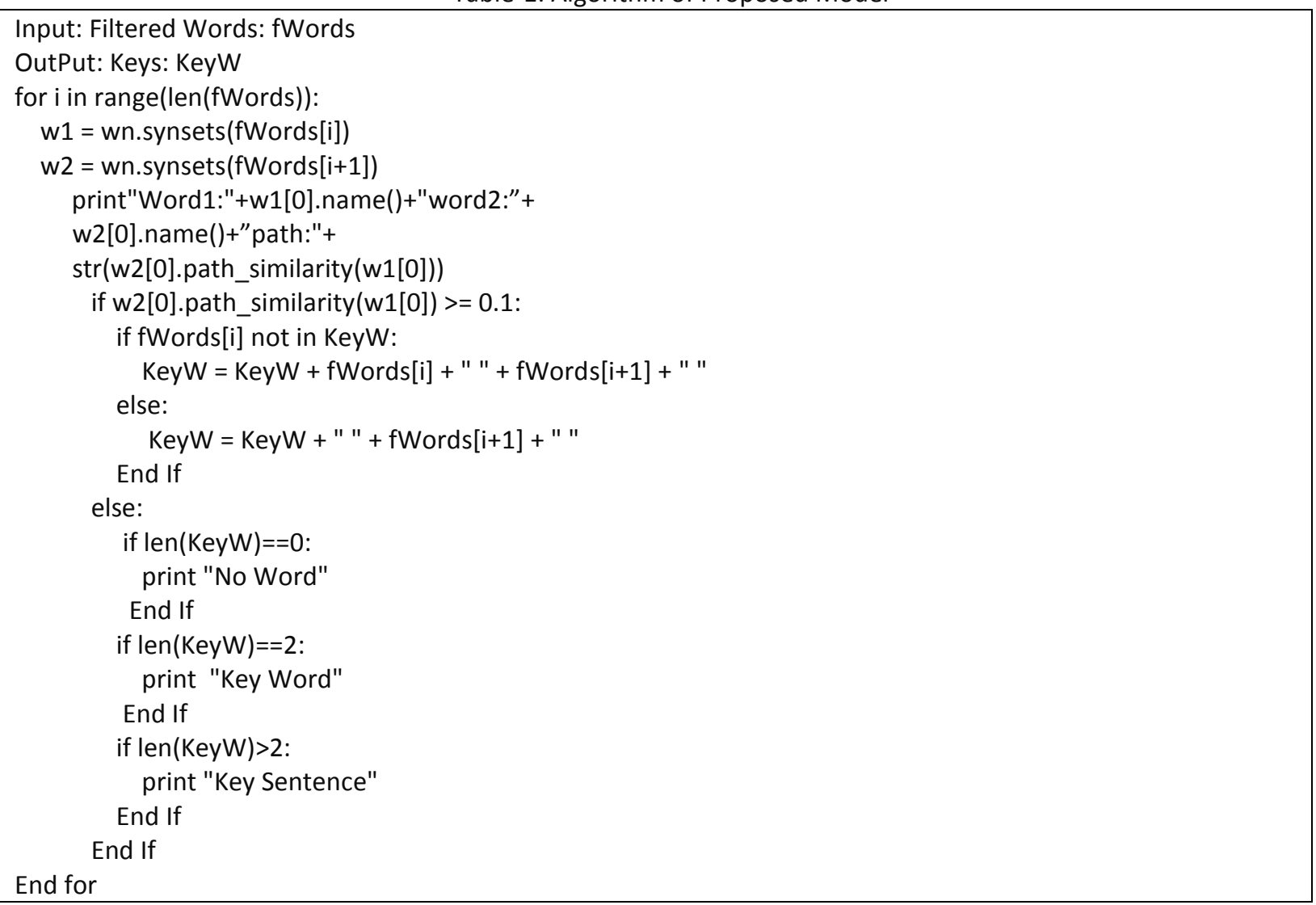

4. Example based on proposed methodology: Here, example containing some text has been applied on proposed work to determine the required words. The contents of text are shown below:

"Around 50 million people are suffering from common mental disorders in Pakistan. The illness afflicts 15 to 35 million adults, which is about ten to twenty percent of the population. Studies show that people with mental illness are much more likely to be a victim than a perpetrator. Unfortunately, there are only 400 trained psychiatrists in the country." 
Extraction of Keywords, Key-Sentence and No-Words has been shown in following figure where each line consists of "Word1, Word2, Distance". If Distance is greater than 0.1, then it will be added to Keys.

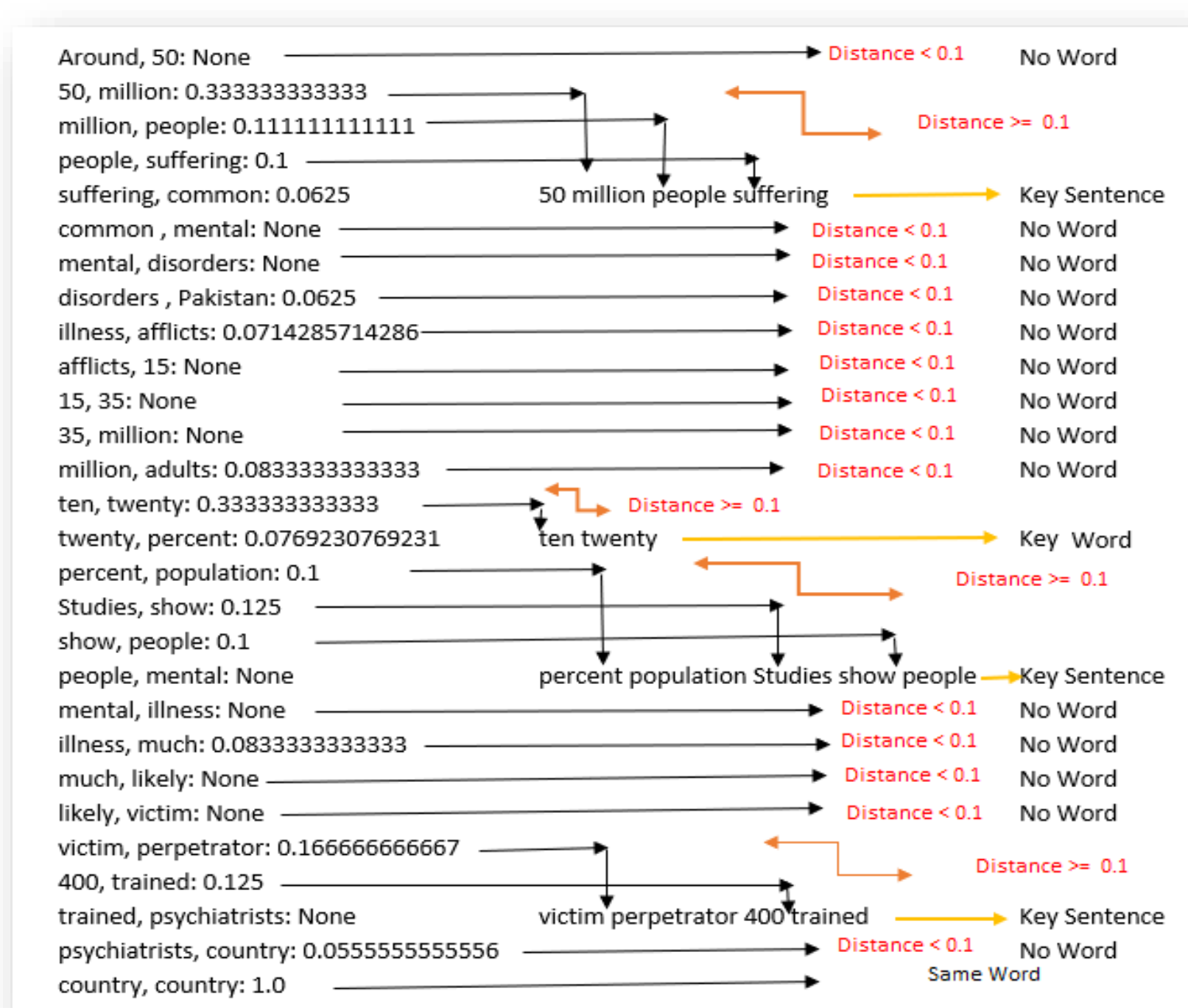

Fig-4: Analyzed Expamle

Following words has been created from above paragraph in inverted comas.

Key Sentence: 50 million people suffering, percent population studies show people, victim, perpetrator 400 trained. Keywords: ten twenty

No word: Remaining all words as single considered as No-Word. Same word will also be considered as No-Word if it is last or it has less than 0.1 distance with next word.

5. Results and Conclusion:- Here we take three documents and a query as text to check LSI score with whole text and using extracted keys through proposed work. These documents are shown in Table-2. Next we will find closest document with query using whole texts and using only keys. 
TABLE-2: SAMPLE OF DOCUMENTS

\begin{tabular}{|l|l|}
\hline Documents & Text \\
\hline Doc-1 & Shipment of gold and diamond damaged destruct and destroy in a fire \\
\hline Doc-2 & Delivery of silver arrived in a silver truck \\
\hline Doc-3 & Shipment of silver and gold arrived in a silver vehicle truck \\
\hline Query & gold silver shipment truck \\
\hline
\end{tabular}

Table-3 is showing the Keys of above said document with the help of similarity distance as said in Fig-1.

TABLE-3: EXTRACTED Keys OF ABOve DOCUMENTS

\begin{tabular}{|c|c|c|}
\hline Documents & Word1, Word2: Distance & Key-Words, Key-Sentences \\
\hline Doc-1 & $\begin{array}{l}\text { Shipment, gold: } 0.0555555555556 \\
\text { gold, diamond: } 0.05 \\
\text { diamond, damaged: } 0.0769230769231 \\
\text { damaged, destruct: } 0.166666666667 \\
\text { destruct, destroy: } 0.5 \\
\text { destroy, fire: None } \\
\text { fire , fire: } 1.0\end{array}$ & 1- damaged destruct destroy \\
\hline Doc-2 & $\begin{array}{l}\text { Delivery, silver: } 0.0666666666667 \\
\text { silver, arrived: } 0.111111111111 \\
\text { arrived, silver: None } \\
\text { silver, truck: } 0.0666666666667 \\
\text { truck, truck: } 1.0\end{array}$ & 1- silver arrived \\
\hline Doc-3 & $\begin{array}{l}\text { Shipment, silver: } 0.0833333333333 \\
\text { silver, gold: } 0.0714285714286 \\
\text { gold, arrived: } 0.0769230769231 \\
\text { arrived, silver: None } \\
\text { silver, vehicle: } 0.0833333333333 \\
\text { vehicle, truck: } 0.2 \\
\text { truck, truck: } 1.0\end{array}$ & 1- vehicle truck \\
\hline Query & $\begin{array}{l}\text { gold, silver: } 0.0714285714286 \\
\text { silver , shipment: } 0.0833333333333 \\
\text { shipment, truck: } 0.1 \\
\text { truck, truck: } 1.0\end{array}$ & 1- shipment truck \\
\hline
\end{tabular}

Here in Doc-1, there is only single key-sentence (damaged destruct destroy), in Doc-2, there is only single key-word (silver arrived) and in Doc-3, there is only single key-word (vehicle truck). Keys of query also a key word as "shipment truck".

Table-4 is showing the LSI scores of each document with respect to query. In 2nd column there is score with whole texts of documents and query. Score of Doc-3 which is highest (0.95), it means Doc-3 is very close to query. Now from 3rd column there is score of each document and query using only extracted keys and here also DOC-3 has 1.0 score means highest score. From both methods DOC-3 is very close to query.

Second method (using keys) of LSI has better performance than first method (using whole text) as we prove in below paragraphs. 
TABLE-4: LSI SCORES OF DOCUMENTS WITH QUERY

\begin{tabular}{|c|c|c|}
\hline Documents & $\begin{array}{c}\text { LSI Score using Whole Text of all } \\
\text { Documents }\end{array}$ & $\begin{array}{c}\text { LSI Score using only keys of } \\
\text { Documents }\end{array}$ \\
\hline Doc-1 & 0.501033 & 0.0 \\
\hline Doc-2 & 0.706893 & -0.01 \\
\hline Doc-3 & 0.951854 & 1.0 \\
\hline
\end{tabular}

In Table-5 processed characters have been identified. From methode-1, LSI will process 67, 44, 61 and 26 characters of documents while from methode-2, it will process only 26,1514 , and 14 characters in memory.

TABle-5: PROCESSED CHARACTERS IN MEMORY

\begin{tabular}{|c|c|c|}
\hline Documents & $\begin{array}{c}\text { No of Characters } \\
\text { Using Whole } \\
\text { Document }\end{array}$ & $\begin{array}{c}\text { No of Characters } \\
\text { Using Only Keys }\end{array}$ \\
\hline Doc-1 & 67 & 26 \\
\hline Doc-2 & 44 & 15 \\
\hline Doc-3 & 61 & 14 \\
\hline Query & 26 & 14 \\
\hline
\end{tabular}

$\mathrm{V}$ matrix and S matrix have same size in method-1 and method-2, while rest of matrix have less size in method-2 i.e. size of matrix $U$ in method-1 is 144 while in method-2 only 49 as shown in following table.

TABLE-6: SIZES OF LSI-MATRICES

\begin{tabular}{|c|c|c|}
\hline LSI-Matrix & $\begin{array}{c}\text { Size using Whole } \\
\text { text of All } \\
\text { Documents }\end{array}$ & $\begin{array}{c}\text { Size using only } \\
\text { Keys of All } \\
\text { Documents }\end{array}$ \\
\hline U & 144 & 49 \\
\hline V & 9 & 9 \\
\hline S & 3 & 3 \\
\hline UK & 24 & 14 \\
\hline Query Matrix & 12 & 7 \\
\hline Feature Matrix & 36 & 21 \\
\hline
\end{tabular}

As actually we have seen that Doc-3 is very close to query and from both methods Doc-3 gets closest distance with respect to query, it means proposed work has correct results. Then we have compared both methods, method-2 got better performance with respect to occupied space and matrices sizes. As there is less size of matrices, so it will take less time to execute.

\section{REFERENCES}

[1] Chinsha, T. C., \& Joseph, S. (2015, February). A syntactic approach for aspect based opinion mining. In Semantic Computing (ICSC), 2015 IEEE International Conference on(pp. 24-31). IEEE.

[2] Qi, L., \& Chen, L. (2011, August). Comparison of model-based learning methods for feature-level opinion mining. In Proceedings of the 2011 IEEE/WIC/ACM International Conferences on Web Intelligence and Intelligent Agent Technology-Volume 01 (pp. 265-273). IEEE Computer Society.

[3] Thakur, D., \& Singh, J. (2015, February). The SAFE miner: A fine grained aspect level approach for resolving the sentiment. In Computer, Communication, Control and Information Technology (C3IT), 2015 Third International Conference on(pp. 1-6). IEEE. 
[4] Poria, S., Cambria, E., Ku, L. W., Gui, C., \& Gelbukh, A. (2014). A rule-based approach to aspect extraction from product reviews. In Proceedings of the second workshop on natural language processing for social media (SocialNLP) (pp. 28-37).

[5] Zhu, J., Wang, H., Tsou, B. K., \& Zhu, M. (2009, November). Multi-aspect opinion polling from textual reviews. In Proceedings of the 18th ACM conference on Information and knowledge management (pp. 17991802). ACM.

[6] Hu, M., \& Liu, B. (2004, August). Mining and summarizing customer reviews. In Proceedings of the tenth ACM SIGKDD international conference on Knowledge discovery and data mining (pp. 168-177). ACM.

[7] Long, C., Zhang, J., \& Zhut, X. (2010, August). A review selection approach for accurate feature rating estimation. In Proceedings of the 23rd International Conference on Computational Linguistics: Posters (pp. 766-774). Association for Computational Linguistics.

[8] Liu, B. (2007). Web data mining: exploring hyperlinks, contents, and usage data. Springer Science \& Business Media.

[9] Marrese-Taylor, E., Velásquez, J. D., \& Bravo-Marquez, F. (2014). A novel deterministic approach for aspectbased opinion mining in tourism products reviews. Expert Systems with Applications, 41(17), 7764-7775.

[10] Gamon, M., Aue, A., Corston-Oliver, S., \& Ringger, E. (2005, September). Pulse: Mining customer opinions from free text. In international symposium on intelligent data analysis (pp. 121-132). Springer, Berlin, Heidelberg.

[11] Zhuang, L., Jing, F., \& Zhu, X. Y. (2006, November). Movie review mining and summarization. In Proceedings of the 15th ACM international conference on Information and knowledge management (pp. 4350). ACM.

[12] Riloff, E., Wiebe, J., \& Wilson, T. (2003, May). Learning subjective nouns using extraction pattern bootstrapping. In Proceedings of the seventh conference on Natural language learning at HLT-NAACL 2003Volume 4 (pp. 25-32). Association for Computational Linguistics.

[13] Zagibalov, T., \& Carroll, J. (2008). Unsupervised classification of sentiment and objectivity in Chinese text. In Proceedings of the Third International Joint Conference on Natural Language Processing: Volume-I.

[14] Jeyapriya, A., \& Selvi, C. K. (2015, February). Extracting aspects and mining opinions in product reviews using supervised learning algorithm. In Electronics and Communication Systems (ICECS), 2015 2nd International Conference on (pp. 548-552). IEEE.

[15] Liu, Q., Gao, Z., Liu, B., \& Zhang, Y. (2013, November). A logic programming approach to aspect extraction in opinion mining. In Web Intelligence (WI) and Intelligent Agent Technologies (IAT), 2013 IEEE/WIC/ACM International Joint Conferences on (Vol. 1, pp. 276-283). IEEE.

[16] Pontiki, M., Galanis, D., Papageorgiou, H., Androutsopoulos, I., Manandhar, S., Mohammad, A. S., ... \& Hoste, V. (2016). SemEval-2016 task 5: Aspect based sentiment analysis. In Proceedings of the 10th international workshop on semantic evaluation (SemEval-2016) (pp. 19-30).

[17] Gupta, D. K., \& Ekbal, A. (2014). IITP: supervised machine learning for aspect based sentiment analysis. In Proceedings of the 8th International Workshop on Semantic Evaluation (SemEval 2014) (pp. 319-323).

[18] Brychcín, T., Konkol, M., \& Steinberger, J. (2014). Uwb: Machine learning approach to aspect-based sentiment analysis. In Proceedings of the 8th International Workshop on Semantic Evaluation (SemEval 2014) (pp. 817-822).

[19] Brun, C., Popa, D. N., \& Roux, C. (2014). Xrce: Hybrid classification for aspect-based sentiment analysis. In Proceedings of the 8th International Workshop on Semantic Evaluation (SemEval 2014) (pp. 838-842).

[20] https://www.ling.upenn.edu/courses/Fall_2003/ling001/penn_treebank_pos.html

[21] Baccianella, S., Esuli, A., \& Sebastiani, F. (2010, May). Sentiwordnet 3.0: an enhanced lexical resource for sentiment analysis and opinion mining. In LREC (Vol. 10, No. 2010, pp. 2200-2204).

[22] Baccianella, S., Esuli, A., \& Sebastiani, F. (2010, May). Sentiwordnet 3.0: an enhanced lexical resource for sentiment analysis and opinion mining. In LREC (Vol. 10, No. 2010, pp. 2200-2204).

[23] S. Saqib, S. M., Mahmood, K., \& Naeem, T. (2016). Comparison of LSI algorithms without and with preprocessing: using text document based search. Accent. Trans. Inf. Security., vol. 1, no. 4, pp. 44-51, 2016.

[24] Phadnis, N., \& Gadge, J. (2014). Framework for document retrieval using latent semantic indexing. International Journal of Computer Applications, 94(14).

[25] Saqib, S. M., Kundi, F. M., \& Ahmad, S. (2018). Unsupervised Learning Method for Sorting Positive and Negative Reviews Using LSI (Latent Semantic Indexing) with Automatic Generated Queries. IJCSNS, 18(1), 56. 